OPEN

SUBJECT AREAS:

PROTEIN ENRICHMENT

SUMOYLATION

STRESS SIGNALLING

ONCOGENES

Received

15 March 2013

Accepted

25 March 2013

Published

22 April 2013

Correspondence and requests for materials should be addressed to M.S.R. (msrodriguez@ inbiomed.org)

\section{Analysis of SUMOylated proteins using SUMO-traps}

\author{
Elisa Da Silva-Ferrada ${ }^{1,2}$, Wendy Xolalpa ${ }^{1,2}$, Valérie Lang ${ }^{2}$, Fabienne Aillet' ${ }^{2}$, Itziar Martin-Ruiz' \\ Carlos F. de la Cruz-Herrera ${ }^{3}$, Fernando Lopitz-Otsoa ${ }^{1}$, Arkaitz Carracedo 1,4, , Seth J. Goldenberg ${ }^{6}$, \\ Carmen Rivas ${ }^{3}$, Patrick England ${ }^{7,8} \&$ Manuel S. Rodríguez ${ }^{1,2}$
}

\begin{abstract}
'Proteomics Unit, CIC bioGUNE, CIBERehd, Bd. 801A, Bizkaia Technology Park, Derio, 48160, Bizkaia, Spain, ${ }^{2}$ Ubiquitylation and Cancer Molecular Biology. Inbiomed, Po Mikeletegi 81, San Sebastián 20009, Gipuzkoa, Spain, ${ }^{3}$ Centro Nacional de Biotecnología, CSIC, Campus Universidad Autónoma de Madrid, Madrid, 28049, Spain, ${ }^{4}$ IKERBASQUE, Basque foundation for science, Bilbao, Spain, ${ }^{5}$ Biochemistry and Molecular Biology Department, University of the Basque Country (UPV/EHU), P. O. Box 644, E-48080 Bilbao, Spain, ${ }^{\circ}$ Progenra Inc., Great Valley Parkway, Malvern, PA 19355, U.S.A., ${ }^{7}$ Institut Pasteur, Proteopole, Plateforme de Biophysique des Macromolécules et de leurs Interactions, ${ }^{8}$ CNRS, UMR3528, 25, rue du Docteur Roux, Paris, 75015 , France.
\end{abstract}

SUMO-modified proteins are recognized by SUMO interacting motifs (SIMs), thus triggering diverse cellular responses. Here SIMs were used to develop SUMO-traps to capture endogenous SUMOylated proteins. Our results show that these small peptides are transferable motifs that maintain their SUMO binding capacity when fused to the heterologous carrier protein GST. The tandem disposition of SIMs increases the binding capacity of SUMO-traps to specifically interact with polySUMO but not poly-Ubiquitin chains. We demonstrate that this SUMO capturing system purifies SUMOylated proteins such as IкB $\alpha$, PTEN, PML or p53 in vitro and in vivo. These properties can be used to explore the many critical functions regulated by protein SUMOylation.

UMO (Small Ubiquitin MOdifier) is an ubiquitin-like protein involved in post- translational modification of critical cellular factors controlling protein localisation, transcription, DNA repair or cell cycle progression among other functions ${ }^{1,2}$. Protein SUMOylation is defined as the covalent modification of target proteins with SUMO molecules on the lysine of the canonical consensus sequence $\psi \operatorname{KxE}$ (where $\psi$ is a large hydrophobic aminoacid and $\mathrm{x}$ any aminoacid) ${ }^{3}$. Generally, this process is carried out by an enzymatic cascade involving three enzymes, E1 (SUMO-activating enzyme (SAE)), E2 (SUMO-conjugating enzyme (Ubc9)) and E3 (SUMO ligating enzyme) $)^{2}$. The reverse reaction known as protein deSUMOylation, is carried out by SENPs or SUSPs (SUMO specific proteases) implicated in the regulation of important cellular responses ${ }^{4-6}$. In mammals three different SUMO molecules are involved in protein SUMOylation: SUMO-1, SUMO-2 and SUMO- $3^{2}$. In response to general cellular insults, such as heat shock, oxidative stress or the use of proteasome inhibitors, the level of protein SUMOylation notably increases ${ }^{7,8}$. Certain viral infections or arsenic trioxide treatment are also able to induce SUMOylation on specific proteins such as p53 and PML (personal communication Rivas C) ${ }^{9,10}$.

Non-covalent interactions with SUMO can also occur through SIMs (SUMO interacting motifs). SIMs are often present within SUMO substrates or enzymes regulating the levels of SUMOylated proteins ${ }^{11-13}$. SIMs were classified in 2000 by Miteva and collaborators in three categories according to their aminoacid residue conservation SIM-a [PILVM]-[ILVM]-x-[ILVM]-[DSE](3); SIM-b [PILVM]-[ILVM]-D-L-T and SIM-r [DSE](3)$[\operatorname{ILVM}]-\mathrm{x}-[\mathrm{ILVMF}](2)^{14,15}$. Generally SIMs contain a hydrophobic core with 3-4 aliphatic residues, adjacent to a negatively charged cluster of acidic amino acids ${ }^{13,16}$. SIMs are present in many key cellular mediators and therefore the identification of new proteins containing SIMs can be important to further understand SUMOdependent regulatory mechanisms $\mathrm{s}^{12,17}$. Particular attention has been given to proteins comprising multiple SIMs, such as CASP8AP2 (CASP8-associated protein 2), RNF4 and RNF111 among others ${ }^{18,15}$. Sun and Hunter (2012) demonstrated that mutation of SIM1 and SIM3 in RNF111 results in the loss of its polySUMO binding capacity. RNF4, the best characterised multiple SIM-containing protein shows SUMO-dependent ubiquitin ligase activity $^{15}$. Among the 4 SIM motifs of RNF4, SIM2 and SIM3 play a more important role in the interaction with polySUMO chains ${ }^{18}$. These findings highlight the importance of clustered SIMs, which efficiently interact with polySUMO chains by increasing the binding capacity for SUMO, since the affinity of an isolated SIM for SUMO is usually low (2-3 $\mu \mathrm{M}$ range $)^{16,13}$. 
Here, we engineered a GST-fusion protein carrying tandem repeats of SIM2 and SIM3 motifs of RNF4 to generate a system to capture SUMOylated proteins. We demonstrate that our SUMOtraps are potent affinity purification tools to isolate total and specific SUMOylated proteins, such as PTEN, I $\mathrm{B} \alpha, \mathrm{PML}$ and $\mathrm{p} 53$ in vivo and/or in vitro.

\section{Results}

Designing SUMO-traps to capture SUMOylated proteins. SUMOtraps, also named SUMO binding entities (SUBEs), were engineered by introducing RNF4-derived SIM2 and SIM3 motifs in tandem, into a GST vector (Fig. 1A). GST itself was modified by introducing an Nterminal His- 6 tag and a C-terminal SV5 epitope (Fig. 1A), allowing to assess the integrity of the constructs (Fig. 1B). The GST system was chosen to obtain a format comparable to the one we used for tandem ubiquitin binding entities (TUBEs) ${ }^{19}$. Two types of SUMO-traps were designed: SUBE-1 (SUBE-long), containing four inserts (eight SIMs), and SUBE-s (SUBE-short), containing only one insert (two SIMs) (Fig. 1A). SUBE-1 was able to pulldown polySUMOylated proteins from HeLa cells challenged at $43^{\circ} \mathrm{C}$ for $1 \mathrm{~h}$ (Fig. 1C) ${ }^{7}$. However, under the same experimental conditions, the efficiency of polySUMOylated protein pulldown by SUBE-s was poor (Fig. 1D). These results support the notion of cooperativity between tandem-repeated SIMs. It has been reported that the acidic aminoacids that flank each SIM core, are important for SUMO recognition ${ }^{11}$. We thus replaced the natural acidic linker between the hydrophobic cores of SIM2 and SIM3 by seven glycine residues. The resulting mutant (SUBE-l mut) is unable to interact with SUMOylated proteins (Fig. 1E), underlining the importance of the flanking acidic aminoacids in the recognition of polySUMO chains.

In addition to their capacity to interact specifically and purify modified proteins, molecular traps such as TUBEs hinder the action of cysteine proteases ${ }^{19}$. To ascertain if this was the case for SUBEs, we tested the capacity of the traps to interfere with the SUMO deconjugation process in a cell free system. Compared to popular cysteine protease inhibitors, such as N-ethylmaleimide (NEM), Iodoacetamide (IAA) and cell-permeable deubiquitylating enzyme (DUB) inhibitor PR-619, SUBEs showed only a modest protection of high molecular weight forms of SUMO-2/-3 compared to the GST control (Supplementary Fig. 1). Although NEM was the most efficient SUMO protease inhibitor (on the basis of free SUMO-2/-3 accumulation), it interferes with GST binding to the glutathione beads (unpublished observations), so we rather used PR-619 in cell media or lysis buffers.

Interaction of SUMO-traps with polySUMO chains. To further demonstrate the cooperativity of tandem SIMs within SUBE-1 when interacting with polySUMO chains, Surface Plasmon Resonance (SPR) experiments were performed using the previously reported tetra-SUMO-2 fusion protein $(4 \mathrm{xS} 2)^{20}$ (Fig. 2). As shown in Fig. 2A, SUBE-1 interacted stronger with $4 \mathrm{xS} 2$ than SUBE-s. GST pulldowns using either SUBE-1 or SUBE-s yielded similar results (Fig. 2B). Of note, no binding of free SUMO-2 (S2) was observed in these assays (Fig. 2B). No significant interaction could be detected between the ubiquitin-trap TUBE-HR23 and $4 \mathrm{xS} 2$, both by SPR (Fig. 2A right panel) and GST pulldown approaches (Fig. 2C),
A
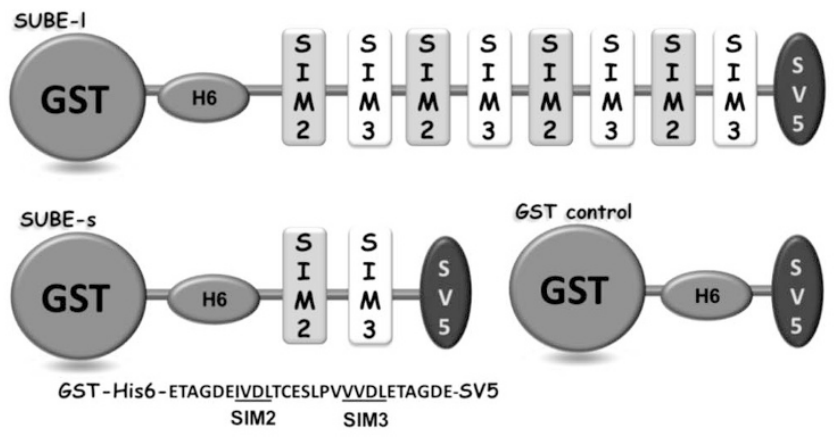

B

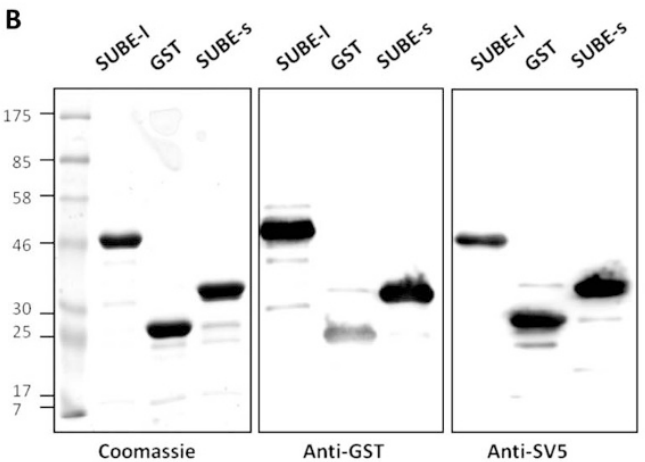

C

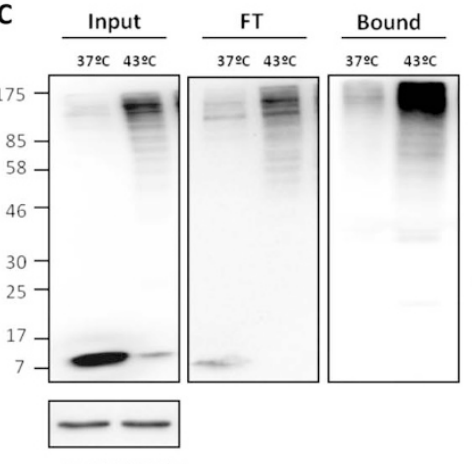

Anti-GAPDH
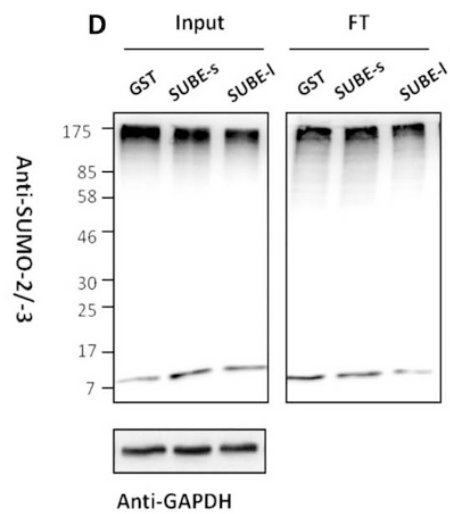
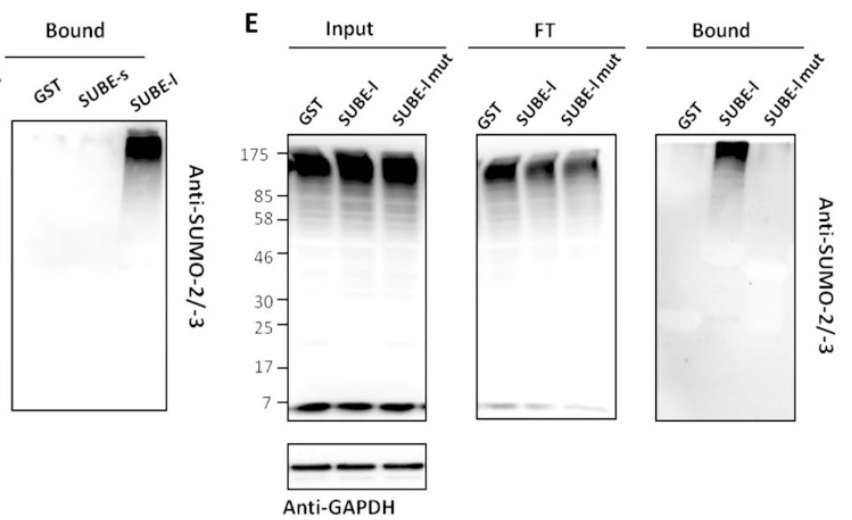

Figure 1 SUMO-binding entities (SUBEs) present the capacity to bind SUMOylated proteins. (A) Cartoon illustrating the design of SUMObinding entities (SUBEs). SUBE-1 and SUBE-s contain 8 and 2 SIMs respectively. The SIM2 and SIM3 region cloned is the natural sequence of RNF4 protein. As a control we used the same GST vector without any insert (GST control). (B) Purified SUBEs can readily be detected by Coomassie, anti-GST and anti-SV5 antibodies. (C) Capture of SUMOylated proteins from heat-shock induced HeLa cells using SUBE-l. (D) SUBE-s do not capture polySUMO proteins from heat-shock induced HeLa cells with the same efficiency as SUBE-l. (E) Contribution of the linker region of SUBE-l to the capture of polySUMO chains from heat-shock induced HeLa cells. In the SUBE-l mutant (SUBE-1 mut), the linker region was mutated to a poly-glycine sequence preserving the length of the natural linker. Western-blot detection with anti-SUMO-2/-3 or GAPDH antibodies. 
A
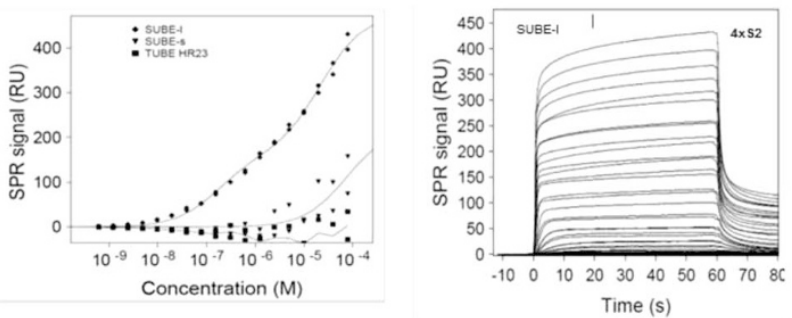

B
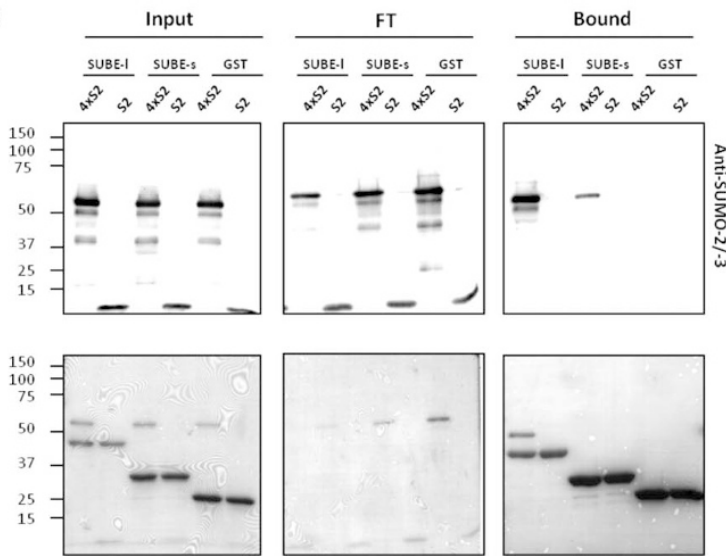

C
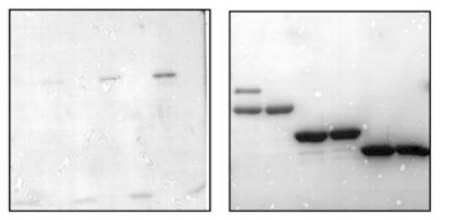

$\mathbf{E}$
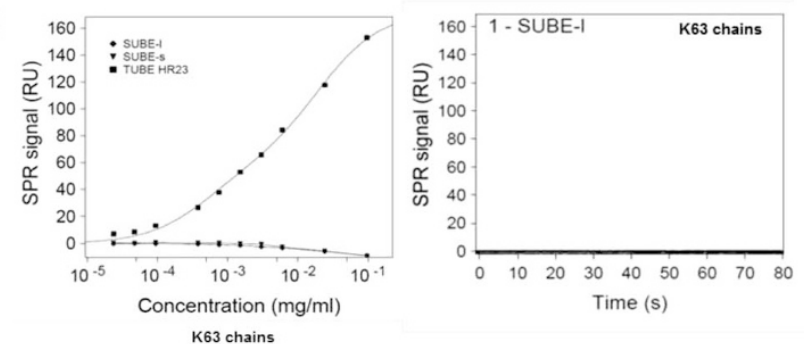
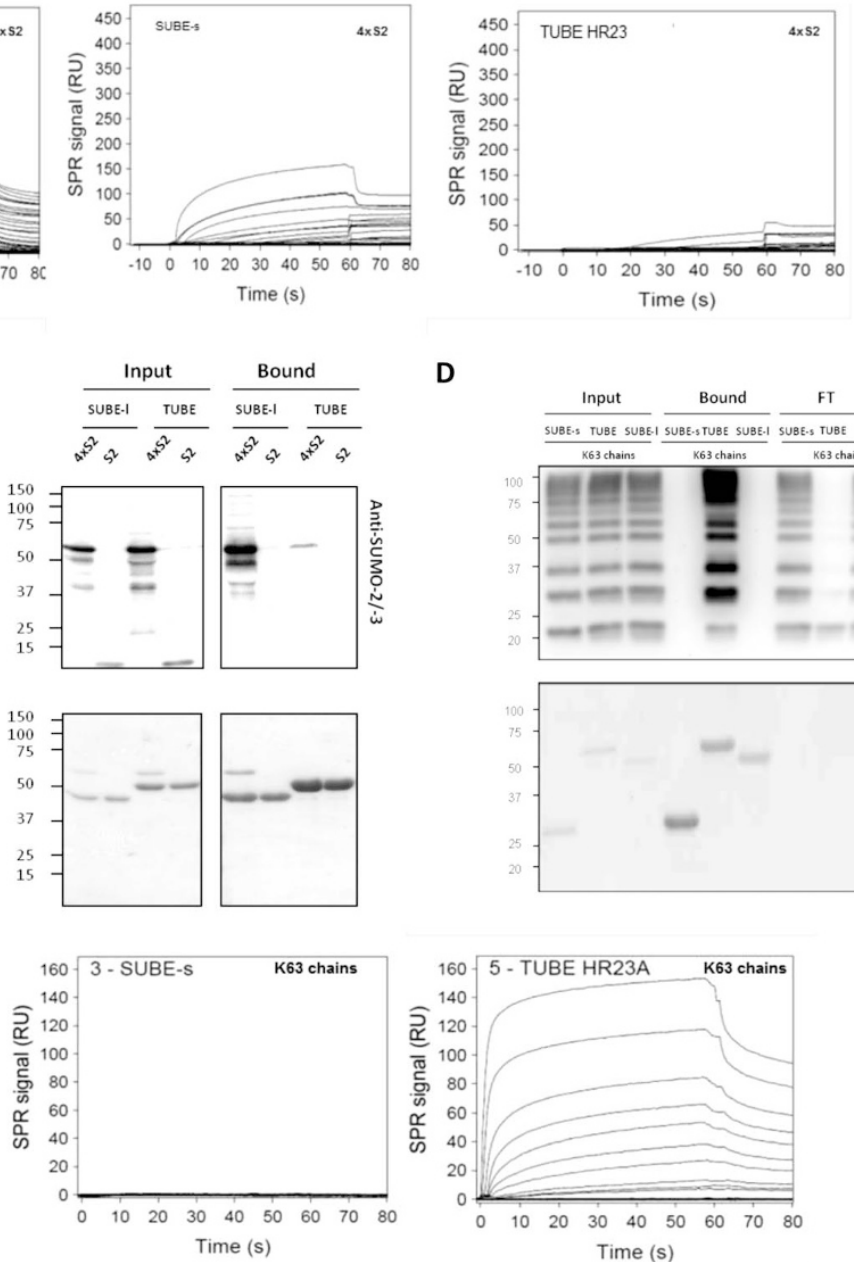

D
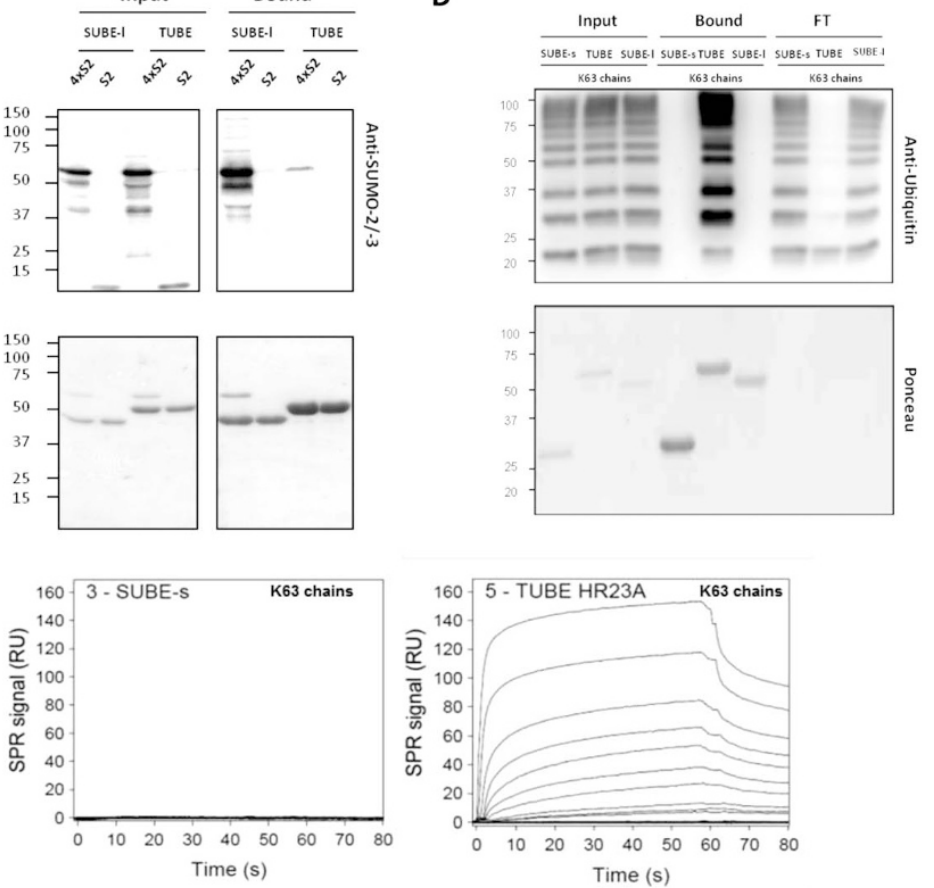

Figure 2 SUBE-1 interacts with polySUMO chains. (A) The interaction of SUMO chains containing four SUMO-2 molecules (4xS2) with SUBE-1, SUBE-s or TUBE-HR23 was monitored by real-time SPR. SUBEs or TUBE-HR23 were captured on an anti-GST surface through their GST moiety, to a level of $100 \mathrm{RU}$, and 4xS2 $(0-150 \mu \mathrm{M})$ was injected in a randomized order. (B) SUBE-l interacts better with 4xS2 chains than SUBE-s. A GST pulldown was performed with both SUBEs to test the interaction with polySUMO chains. Input, flow-through (FT) and bound fractions were analysed by Westernblot against anti-SUMO-2/-3 antibody. (C-D) Specific interaction of SUBE-1 with 4xS2 chains. (C) A similar approach than (B) was used to investigate if the 4xS2 was also able to bind other molecular traps such as TUBEs. (D) Conversely it was also tested if SUBEs were able to bind a collection of K63 ubiquitin chains. Western-blot detection with the indicated antibodies. (E) The absence of binding of both SUBE-1 and SUBE-s to K63 ubiquitin chains was verified by SPR in the same conditions as (A). TUBE-HR23 was used as positive control.

showing that polySUMO chains are recognized specifically by our SUBE-l trap. The specificity of SUBEs was further investigated using linear K63 poly-ubiquitin chains (and TUBE-HR23 as a positive control). GST pulldowns (Fig. 2D) and SPR assays (Fig. 2E) showed that none of the SUBEs could interact with K63 chains, unlike TUBE-HR23. Altogether, these experiments demonstrate that SUBEs specifically interact with polySUMO chains and do not bind to K63 poly-ubiquitin chains and free SUMO moieties.

As the SUMO-2 moieties of $4 \mathrm{xS} 2$ construct are linked by peptide bonds, the capacity of SUBE-1 to interact with polySUMO chains linked by isopeptide bonds was tested using an in vitro SUMOylation assay (Supplementary Fig. 2). This assay, with no added substrate and SUMO E3 enzyme, resulted in a modest production of polySUMO chains. However, it can be seen that chains containing multiple molecules of SUMO-2/3 are efficiently depleted from the extract by SUBE-1 and recovered in the bound fraction (Supplementary Fig. 2). Chains comprising a combination of SUMO-2 and SUMO-3 molecules were more efficiently retained than those containing only SUMO-1 (Supplementary Fig. 2). Thus, these results indicate that SUBE-l binds to isopeptide bond linked polySUMO chains in a similar way than to the $4 \mathrm{xS} 2$ construct.
Capture of multiple SUMO substrates using SUMO-traps. To verify that SUBE-1 could be used to purify SUMOylated substrates, 4 different subtrates: $\mathrm{PML}^{10}$, IкB $\alpha^{21,22}, \mathrm{p} 53^{9}$ and $\mathrm{PTEN}^{23}$ were SUMOylated in vitro using SUMO-1 or SUMO-2/-3 (Fig. 3 A-D). PolySUMOylated forms of all these substrates were efficiently retained by SUBE-1 only when SUMO-2/-3 was used in the reaction, SUMO-1 conjugated forms being captured much less efficiently (Fig. $3 \mathrm{~A}-\mathrm{D}$ ). In our experimental conditions, the GST control bound to unmodified sticky forms of proteins such as IкB $\alpha$ or p53 in different proportions (Supplementary Fig. 3). Given that SUMO- 1 and SUMO-2/-3 poly-SUMOylated chains were present in similar quantities in the input of each experimental set (Fig. $3 \mathrm{~A}-\mathrm{D}$ ) we can conclude that SUBE-l preferentially captures substrates comprising polySUMO-2/-3 chains.

SUMO-traps captures SUMOylated PML and p53 in vivo. To investigate the capacity of the SUMO-trap SUBE-l to interact with naturally SUMOylated PML or p53, SUMOylation was induced by challenging/treating cells with arsenic trioxide (ATO), (interferon- $\alpha$ ) or vesicular stomatitis virus (VSV) infection as indicated (Fig. 4 AD). Conditions were set to capture SUMOylated PML from NB4 
A

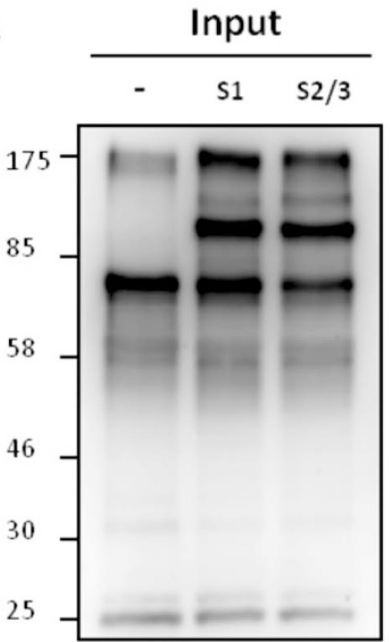

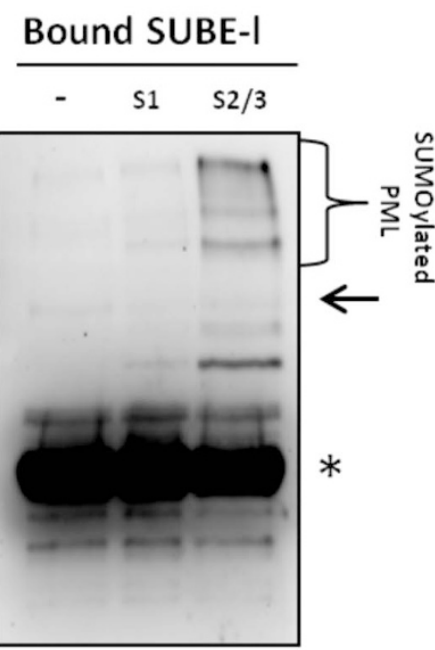

Anti-PML

C
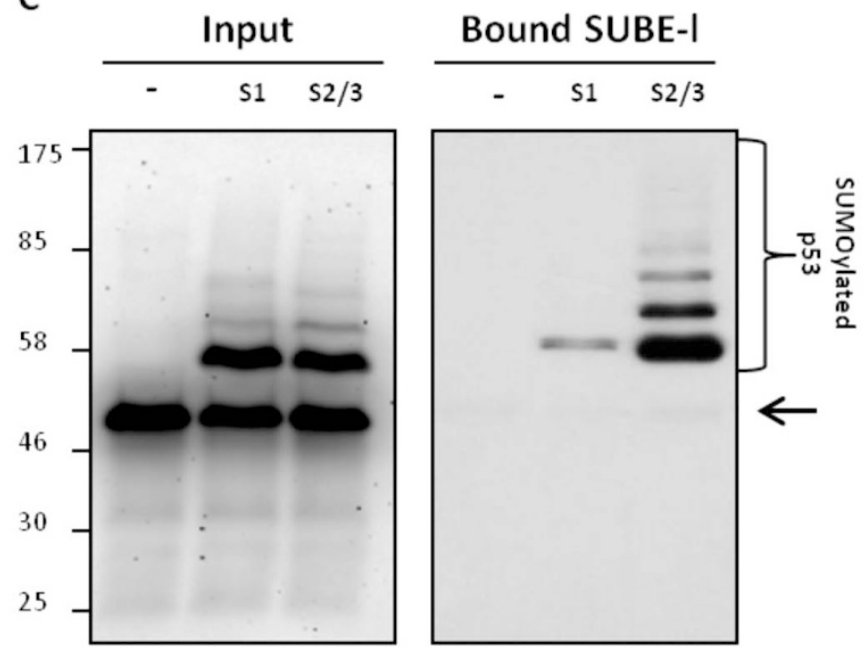

B

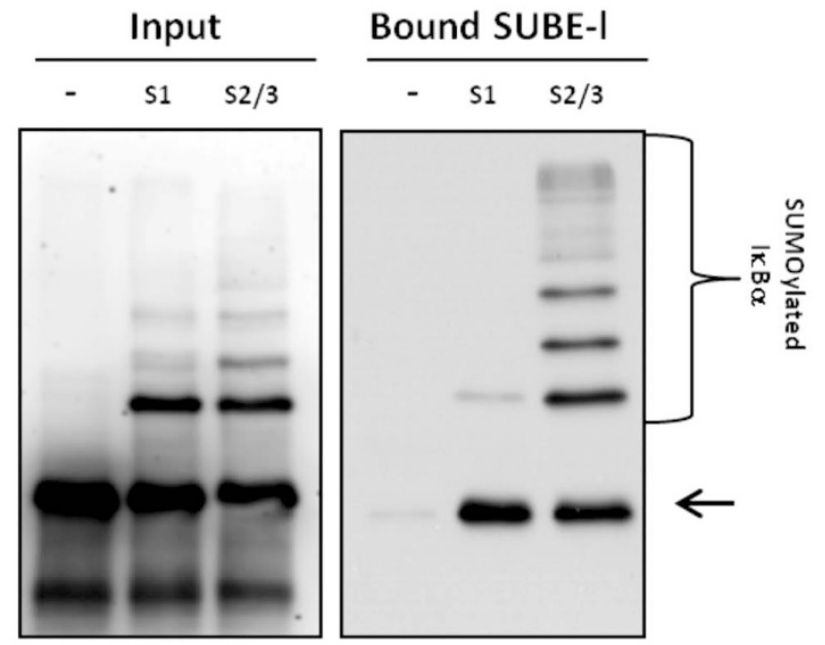

Anti-IKB $\alpha$

D

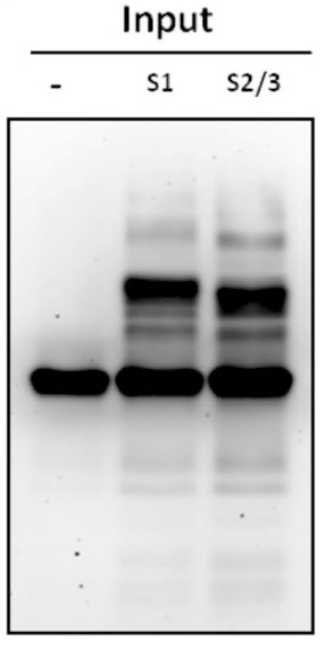

\section{Bound SUBE-I}

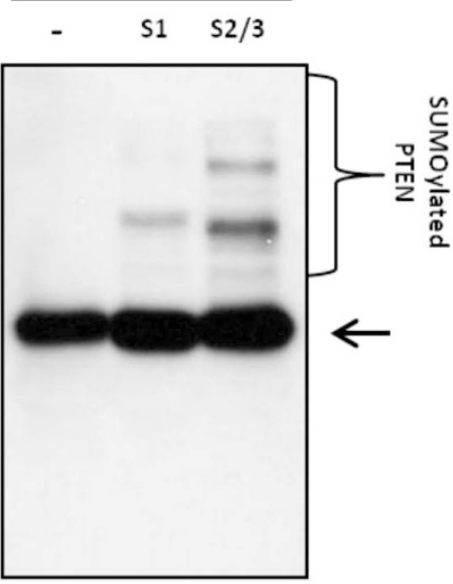

Anti-p53

\section{Anti-PTEN}

Figure 3 Multiple SUMO substrates can be captured by SUBE-l. SUMOylated (A) PML, (B) I $\kappa$ B $\alpha,(\mathrm{C})$ p53 and (D) PTEN were SUMOylated in vitro with SUMO-1 (S1) or SUMO-2/-3 (S2/3) to generate modified proteins (input). Control reactions (-) do not contain SUMO activating enzyme. SUBE-1 captured proteins are present in the bound fraction. Analysis by Western-blot using the indicated antibodies. The arrow shows unmodified proteins.

* non specific signal.

(acute promyelocytic leukemia cell line, Fig. 4A) and MCF7 cells (breast cancer cell line, Fig. 4B), and to evaluate the co-localization of SUMO-2/-3 and PML in the nuclear bodies of NB4 cells (Fig. 4C). One hour pre-treatment with proteasome inhibitor MG-132 followed by one hour of stimulation with ATO resulted in the optimal capture of SUMOylated PML (Fig. 4A and B). Basal levels of SUMOylated PML were not captured indicating that this is an inducible process ${ }^{10}$. To capture SUMOylated p53, HEK-293 transfected with His6SUMO-2 were treated with IFN- $\alpha$ for 24 hours or infected with VSV for $4 \mathrm{~h}$ (Fig. 4D). Here again, SUMOylated forms of p53 were captured and detected by Western blot only when cells were induced. The importance of SUMOylated p53 induced by IFN- $\alpha$ or VSV infection was further studied by Marcos-Villar et al (Rivas C. personal communication).

\section{Discussion}

As SIMs are very short peptide sequences, their efficiency for SUMOylated protein purification could be questioned, especially in terms of affinity and specificity. The multiplication of SIM motifs to increase the capacity to interact with polySUMOylated proteins is an approach naturally used by the cell in proteins such as RNF4 and RNF $111^{18,15}$. Here we show that small SIM sequences can be transferred to non-related proteins, resulting in SUMO-binding entities (SUBEs) that have the capacity to bind and even purify SUMOylated proteins. Our finding opens the door to the design of multiple traps using different SIMs to study SUMO-regulated events. More studies are warranted to ascertain whether the nature of SIM sequences will determine the type of SUMOylated proteins captured. However, it is tempting to speculate that the flanking acidic regions around the hydrophobic core in the SUMO-binding domain could provide sufficient sequence elements to distinguish between different SUMOchain types. In our experimental conditions, not all SUMOylated proteins were captured from cell extracts using SUBE-l (Fig. 1C-E and Fig. 4 B, C). Increasing the concentration of SUBE-l did not widen its selectivity towards other SUMOylated proteins, indicating that the non-captured material had SUMO chains with a different 
A

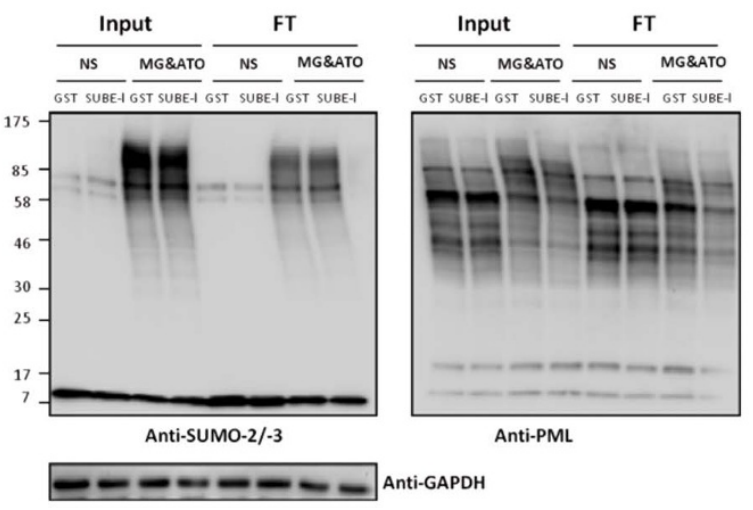

Bound

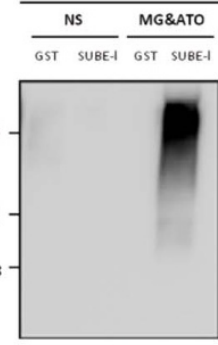

Anti-SUMO-2/-3 NS MG\&ATO

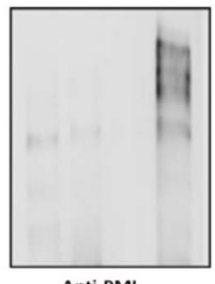

Anti-PML

C
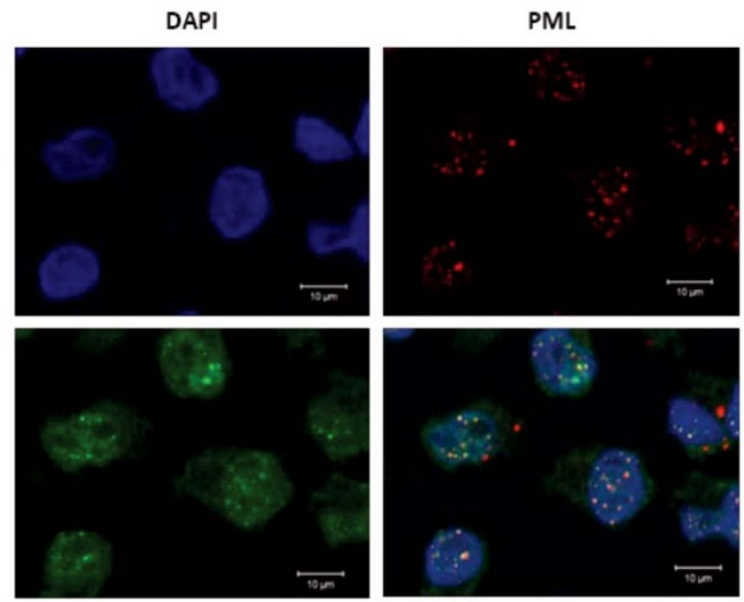

SUMO-2/3
B
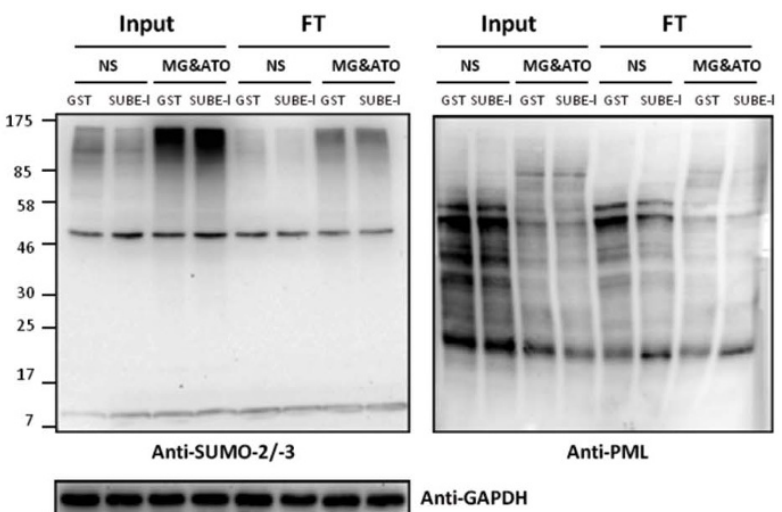

Anti-GAPDH

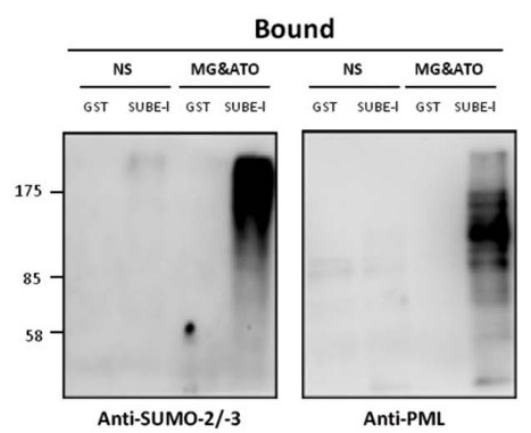

D

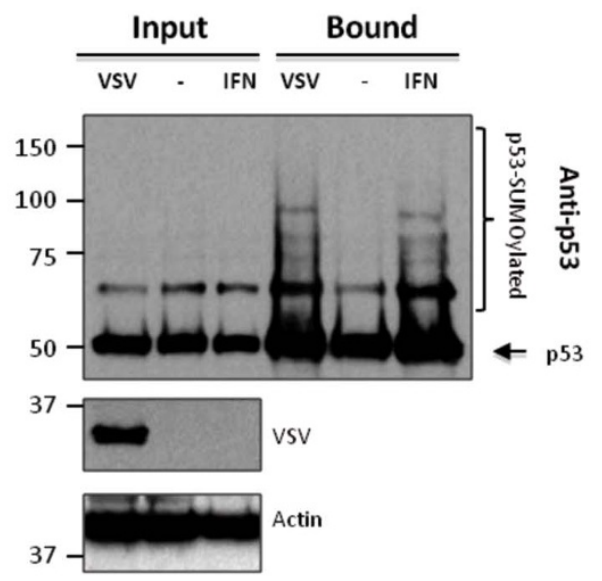

Figure 4 Capture of SUMOylated PML and p53 using SUBE-1. NB4 cells (A) or MCF7 (B) were pre-treated with MG-132 before 1 h of ATO treatment. (C) SUMO and PML can be co-localized in the nuclear bodies (NB) of NB4 cells under the same conditions than (A or B). SUMOylated p53 can be captured by SUBE-1 (D). HEK-293 were untreated or treated with IFN- $\alpha$ or infected with VSV. Input, flow-through (FT) and bound fractions were analysed with anti-SUMO-2/-3, PML or p53 antibodies. GAPDH (glyceraldehyde 3-phosphate dehydrogenase) was used as loading control.

architecture. The natural domains of RNF4 have already been used as affinity traps to purify SUMOylated proteins or to identify putative SUMOylated substrates by mass spectrometry ${ }^{20}$. By extending the number of SIMs, our SUBEs show an improved capacity to purify SUMOylated proteins compared to the natural RNF4 SIM sequences (Supplementary Fig. 4). The use of molecular traps such as SUBEs to analyse endogenous SUMOylated proteins will be critical to understand the role of this post translational modification in the control of diverse fundamental molecular events.

\section{Methods}

Cloning, protein expression and purification. Synthetic oligonucleotides encoding the SIM2 and SIM3 regions of RNF4 (P78317) (41 to 63:ETAGDEIVDLTCESLEPV VVDL) were cloned in tandem into the PGEX-6P1vector (Amersham). This vector was modified in frame by the introduction, of a His6 tag and a SV5 epitope upstream and downstream of the Multi-Cloning Site, respectively ${ }^{19}$. Such modifications allow the recognition of the construct by the respective anti-His6 and anti-SV5 antibodies. SUBEs (produced by Inbiomed Pharma) were expressed in Escherichia coli BL-21 strain and purified by affinity chromatography using GST agarose beads (Glutathione S-transferase, Biontex), and ion exchange chromatography, using Sepharose beads (Sigma), according to manufacturers' instructions.

Protein pulldown. In order to use SUBEs as affinity traps for total and specific SUMOylated protein pulldown, different cell lines such as NB4, HeLa and MCF-7 were used. Cells were grown at $37^{\circ} \mathrm{C}$ in RPMI (NB4 cells) or DMEM (HeLa, MCF-7 and HEK-293) media (Gibco) both supplemented with 10\% FBS. In the case of HeLa, $2 \times 10^{6}$ cells were treated 30 minute with $20 \mu \mathrm{M} \mathrm{MG}-132$, (Sigma) and stressed for 60 minutes at $43^{\circ} \mathrm{C}$ (to induce SUMOylation) ${ }^{7}$. For NB4 and MCF-7, $5 \times 10^{6}$ and $2 \times 10^{6}$ cells, respectively, were plated and treated next day for 1 hour with $20 \mu \mathrm{M}$ MG-132, and stressed for 1 hour by 0,15 $\mu$ M Arsenic Trioxide (ATO) (Sigma). HEK293 were transfected with His6-SUMO-2 protein and were treated with $500 \mathrm{U} / \mathrm{ml}$ of IFN- $\alpha$ (GenScript) for $24 \mathrm{~h}$, infected with Indiana strain VSV at an MOI of $1 \mathrm{PFU} / \mathrm{ml}$ for $4 \mathrm{~h}$, or left untreated. After all treatments, cells were sonicated twice for 
15 seconds with $10 \%$ amplitude (Branson digital sonifier) in $500 \mu \mathrm{l}$ of lysis buffer (50mM Tris pH 8.5; $150 \mathrm{mM} \mathrm{NaCl}, 5 \mathrm{mM} \mathrm{EDTA,} 1 \%$ Igepal, supplemented with $1 \times$ protease inhibitor cocktail (Roche) and $50 \mu \mathrm{M}$ of PR-619 (ubiquitin and ubiquitinlike isopeptidases inhibitor, LifeSensors).

Lysates were centrifuged at 14000xg (Beckman Coulter Microfuge 22R) and the supernatant was incubated with $50 \mu \mathrm{l}$ of GST-agarose beads containing $50 \mu \mathrm{g}$ of SUBEs or GST and $1 \mathrm{mM}$ DTT (Dithiothreitol) for $2 \mathrm{~h}$ at $4^{\circ} \mathrm{C}$. Beads were then pulled down by centrifugation, 1000xg for 5 minutes (Beckman Coulter Microfuge 22R), and $1 / 10$ of the unbound fraction was saved for western blot analysis (flow throughFT). Washes were carried out using 30 column volumes of wash buffer (50 mM Tris $\mathrm{pH} 8.5 ; 50 \mathrm{mM} \mathrm{NaCl}, 5 \mathrm{mM}$ EDTA and 1\% Igepal). Elutions were performed in one column volume of $2 \times$ Laemmli Buffer.

For western blot analysis, samples were separated in $10 \%$ polyacrylamide gels and membranes were incubated with anti-PML (Bethyl Laboratories Inc), anti-p53 (DO1, Santa Cruz), anti-IкB $\alpha$ (Cell signalling), anti-PTEN (Cell signalling) and antiSUMO-1 or anti-SUMO-2/-3 (Eurogentec) antibodies.

Immunofluorescence assays. The day before the experiment, $3 \times 10^{5} \mathrm{MCF}-7$ cells and $1 \times 10^{6} \mathrm{NB} 4$ cells were plated in a 24 well plate. Cells were treated with $20 \mu \mathrm{M}$ MG-132 for $1 \mathrm{~h}$, and 0,15 $\mathrm{MM}$ ATO for an additional hour. Cells were then washed with PBS 1X, fixed with 1\% paraformaldehyde and permeabilized in PBS 1X/Triton $0,1 \%$. Direct immunofluorescence measurements were performed as previously reported ${ }^{24}$. Monoclonal SUMO-1 and SUMO-2 antibodies (generously provided by C. Gwizdek and M. Matunis) were used at a final dilution of $1 / 50$ and rabbit polyclonal PML (Bethyl Laboratories Inc) antibody was used at 1/500. Texas Red and Alexa 288 were respectively used as secondary antibodies, at 1/1000 dilution.

In vitro SUMOylation assay. For the SUMOylation assays, in vitro transcribed/ translated IкB $\alpha$, PML or $\mathrm{p} 53$, recombinant PTEN $(50 \mathrm{ng} / \mathrm{pt})$ were incubated in a buffer containing an ATP regenerating system [(50 mM Tris pH 7.5, $10 \mathrm{mM} \mathrm{MgCl}$, $2 \mathrm{mM}$ ATP, $10 \mathrm{mM}$ creatine phosphate (Sigma), $3.5 \mathrm{U} / \mathrm{ml}$ of creatine kinase (Sigma), and $0.6 \mathrm{U} / \mathrm{ml}$ of inorganic pyrophosphatase (Sigma)], $10 \mu \mathrm{g}$ of SUMO- $1,-2$ or -3 or $5 \mu \mathrm{g}$ (if used in combination with SUMO-2 and SUMO-3), Ubc9 $(0.325 \mu \mathrm{g})$ and purified SAE1/2 ( $0.8 \mu \mathrm{g}$, ENZO Life Sciences). For the formation of SUMO-1 or -2/-3 chains (Supplementary fig. 2), no protein substrate was added to the previously described assay. Reactions were incubated at $30^{\circ} \mathrm{C}$ for 2 hours and stopped by addition of SDS sample buffer. For pulldown assays, 1/10 of input was saved and the rest of the reaction was incubated with $50 \mu \mathrm{l}$ of GST-agarose beads containing $50 \mu \mathrm{g}$ of SUBEs or GST and $1 \mathrm{mM}$ DTT (Dithiothreitol) for $2 \mathrm{~h}$, at $4^{\circ} \mathrm{C}$. After incubation, beads were pulled down by centrifugation, as previously described, and 1/10 of the unbound fraction (FT) was kept for western analysis. Subsequently, the same beads were washed with 30 column volumes of binding buffer $(50 \mathrm{mM}$ Tris pH $8.5 ; 50 \mathrm{mM}$ $\mathrm{NaCl}, 5 \mathrm{mM}$ EDTA and 1\% Igepal) and were resuspended in one column volume of $2 \times$ Laemmli Buffer.

Surface Plasmon Resonance experiments. SPR experiments were performed on a Biacore 3000 system, equilibrated at $25^{\circ} \mathrm{C}$ in HBS-EP buffer $(0.01 \mathrm{M}$ HEPES pH 7.4, $0.15 \mathrm{M} \mathrm{NaCl}, 3 \mathrm{mM}$ EDTA, $0.005 \%$ surfactant P20) (GE Healthcare), using a CM5 sensor chip with a density of around 6000 resonance units (RU) of covalently immobilized anti-GST antibody (GE Healthcare). Approximately $100 \mathrm{RU}$ of SUBEs [SUBE-1 (1-long) and SUBE-s (s-short)] were captured by the antibody. The 4xS2 (4xSUMO-2) molecule (generously provided by R.T. Hay ${ }^{20}$, and the multi-ubiquitin chains (Ub2-7, K63 chains, Enzo) were injected for 60s at a flow rate of $30 \mu \mathrm{l} / \mathrm{min}$. All injections were carried out in duplicate and in randomized order.

1. Garcia-Dominguez, M. \& Reyes, J. C. SUMO association with repressor complexes, emerging routes for transcriptional control. Biochimica et biophysica acta 1789, 451-9 (2009).

2. Sarge, K. D. \& Park-Sarge, O.-K. Chapter Four - SUMO and Its Role in Human Diseases. Volume 288, 167-183 (2011).

3. Rodriguez, M. S., Dargemont, C. \& Hay, R. T. SUMO-1 conjugation in vivo requires both a consensus modification motif and nuclear targeting. The Journal of biological chemistry 276, 12654-9 (2001)

4. Mikolajczyk, J. et al. Small ubiquitin-related modifier (SUMO)-specific proteases: profiling the specificities and activities of human SENPs. The Journal of biological chemistry 282, 26217-24 (2007).

5. Drag, M. \& Salvesen, G. S. DeSUMOylating enzymes--SENPs. IUBMB life 60 , 734-42 (2008).

6. Hickey, C. M., Wilson, N. R. \& Hochstrasser, M. Function and regulation of SUMO proteases. Nat Rev Mol Cell Biol 13, 755-766 (2012).

7. Golebiowski, F. et al. System-wide changes to SUMO modifications in response to heat shock. Science signaling 2, ra24 (2009).
8. Častorálová, M. et al. SUMO-2/3 conjugates accumulating under heat shock or MG132 treatment result largely from new protein synthesis. Biochimica et Biophysica Acta (BBA) - Molecular Cell Research 1823, 911-919 (2012).

9. Rodriguez, M. S. et al. SUMO-1 modification activates the transcriptional response of $\mathrm{p} 53$. The EMBO journal 18, 6455-61 (1999).

10. Lallemand-Breitenbach, V. et al. Arsenic degrades PML or PML-RARalpha through a SUMO-triggered RNF4/ubiquitin-mediated pathway. Nature cell biology 10, 547-55 (2008)

11. Matic, I. et al. Site-specific identification of SUMO-2 targets in cells reveals an inverted SUMOylation motif and a hydrophobic cluster SUMOylation motif. Molecular cell 39, 641-52 (2010).

12. Song, J., Durrin, L. K., Wilkinson, T. a, Krontiris, T. G. \& Chen, Y. Identification of a SUMO-binding motif that recognizes SUMO-modified proteins. Proceedings of the National Academy of Sciences of the United States of America 101, 14373-8 (2004).

13. Hecker, C.-M., Rabiller, M., Haglund, K., Bayer, P. \& Dikic, I. Specification of SUMO1- and SUMO2-interacting motifs. The Journal of biological chemistry 281, 16117-27 (2006)

14. Miteva, M., Keusekotten, K., Hofmann, K., Praefcke, G. J. K. \& Dohmen, R. J. CHAPTER 16 SUMOYLATION AS A SIGNAL FOR POLYUBIQUITYLATION AND PROTEASOMAL DEGRADATION (C) 2010 Copyright Landes Bioscience. Not for Distribution. 195-214 (2010).

15. Sun, H. \& Hunter, T. Poly-small ubiquitin-like modifier (PolySUMO)-binding proteins identified through a string search. The Journal of biological chemistry 287, 42071-83 (2012).

16. Kerscher, O. SUMO junction-what's your function? New insights through SUMO-interacting motifs. EMBO reports 8, 550-5 (2007).

17. Lin, D.-Y. et al. Role of SUMO-interacting motif in Daxx SUMO modification, subnuclear localization, and repression of sumoylated transcription factors. Molecular cell 24, 341-54 (2006).

18. Tatham, M. H. et al. RNF4 is a poly-SUMO-specific E3 ubiquitin ligase required for arsenic-induced PML degradation. Nature cell biology 10, 538-46 (2008).

19. Hjerpe, R. et al. Efficient protection and isolation of ubiquitylated proteins using tandem ubiquitin-binding entities. EMBO reports 10, 1250-8 (2009).

20. Bruderer, R. et al. Purification and identification of endogenous polySUMO conjugates. EMBO reports 12, 142-8 (2011).

21. Desterro, J. M., Rodriguez, M. S. \& Hay, R. T. SUMO-1 Modification of IкB $\alpha$ Inhibits NF-kB Activation. Molecular Cell 2, 233-239 (1998).

22. Aillet, F. et al. Heterologous SUMO-2/3-Ubiquitin Chains Optimize IкB $\alpha$ Degradation and NF-KB Activity. PloS one 7, e51672 (2012).

23. González-Santamaría, J. et al. Regulation of the tumor suppressor PTEN by SUMO. Cell death \& disease 3, e393 (2012).

24. Midgley, C. a et al. An N-terminal p14ARF peptide blocks Mdm2-dependent ubiquitination in vitro and can activate p53 in vivo. Oncogene 19, 2312-23 (2000).

\section{Acknowledgements}

We would like to thank C. Gwizdek, M. Matunis and R.T. Hay for reagents and tools provided. This work was funded by the MINECO (Spain) grant BFU2008-01108/BMC and BFU2011-28536 (MSR), Department of Industry of the Government of the Basque Country (Etortek Research Programmes 2011/2012). Our group at Inbiomed is supported by the "Obra Social KUTXA" and the Diputación Foral de Guipúzcoa. E.DS-F is fellow from Fundação para a Ciência e Tecnologia, Ministério da Educação e Ciência-Portugal, Grant SFRH/BD/71514/2010.

\section{Author contributions}

E.D.S.-F., W.X., V.L., F.A., I.M., F.L.-O., C.F.C.H. and M.S.R. carried out experiments; A.C., S.J.G., C.R., P.E. provided material, technical support and know-how; E.D.S.-F., M.S.R. wrote the manuscript. All authors designed experiments, discussed results and commented on the manuscript.

\section{Additional information}

Supplementary information accompanies this paper at http://www.nature.com/ scientificreports

Competing financial interests: This work is part of a patent application by CIC bioGUNE

License: This work is licensed under a Creative Commons

Attribution-NonCommercial-ShareALike 3.0 Unported License. To view a copy of this license, visit http://creativecommons.org/licenses/by-nc-sa/3.0/

How to cite this article: Da Silva-Ferrada, E. et al. Analysis of SUMOylated proteins using SUMO-traps. Sci. Rep. 3, 1690; DOI:10.1038/srep01690 (2013). 